\title{
On a state space approach to nonlinear $H_{\infty}$ control
}

\section{A.J. van der Schaft}

Dept. of Applied Mathematics, University of Twente, P.O. Box 217, 7500 AE Enschede, The Netherlands

\section{Received 18 August 1990}

Abstract: We study the standard $H_{\infty}$ optimal control problem using state feedback for smooth nonlinear control systems. The main theorem obtained roughly states that the $L_{2}$-induced norm (from disturbances to inputs and outputs) can be made smaller than a constant $\gamma>0$ if the corresponding $H_{\infty}$ norm for the system linearized at the equilibrium can be made smaller than $\gamma$ by linear state feedback. Necessary and sufficient conditions for the latter problem are by now well-known, e.g. from the state space approach to linear $H_{\infty}$ optimal control. Our approach to the nonlinear $H_{\infty}$ optimal control problem generalizes the state space approach to the linear $H_{\infty}$ problem by replacing the Hamiltonian matrix and corresponding Riccati equation as used in the linear context by a Hamiltonian vector field together with a Hamilton-Jacobi equation corresponding to its stable invariant manifold.

AMS Subject Classifications: 93C05, 58F05, $49 \mathrm{C} 05$.

Keywords: Nonlinear $H_{\infty}$ control; state feedback; $L_{2}$-induced norm; linearization; hyperbolic Hamiltonian vector fields; stable manifolds; Lagrangian submanifolds; Hamilton-Jacobi equation.

\section{Introduction}

Consider a smooth (i.e. $C^{\infty}$ ) nonlinear system (see $[10,12])$

$$
\begin{gathered}
\dot{x}=f(x)+\sum_{j=1}^{m} g_{j}(x) u_{j}, \\
u=\left(u_{1}, \ldots, u_{m}\right) \in \mathbb{R}^{m}, \\
y_{j}=h_{j}(x), \quad j=1, \ldots, p, \\
y=\left(y_{1}, \ldots, y_{p}\right) \in \mathbb{R}^{p},
\end{gathered}
$$

where $x=\left(x_{1}, \ldots, x_{n}\right)$ are local coordinates for the smooth state space manifold $M$. We assume that $x_{0} \in M$ is an equilibrium, i.e. $f\left(x_{0}\right)=0$, and without loss of generality we assume that $h_{j}\left(x_{0}\right)$
$=0, j=1, \ldots, p$. For simplicity of notation we will throughout abbreviate (1) as

$\dot{x}=f(x)+g(x) u, \quad u \in \mathbb{R}^{m}, y \in \mathbb{R}^{p}, x \in M$,

$y=h(x), \quad f\left(x_{0}\right)=0, h\left(x_{0}\right)=0$,

with $g(x)$ the $n \times m$ matrix with $j$-th column $g_{j}(x)$. Furthermore we consider the linearization of (2) around $x_{0}$, denoted as

$\dot{\bar{x}}=F \bar{x}+G \bar{u}, \quad \bar{u} \in \mathbb{R}^{m}, \bar{x} \in \mathbb{R}^{n}$,

$\bar{y}=H \bar{x}, \quad \bar{y} \in \mathbb{R}^{p}$,

with

$F=\frac{\partial f}{\partial x}\left(x_{0}\right), \quad G=g\left(x_{0}\right), \quad H=\frac{\partial h}{\partial x}\left(x_{0}\right)$.

Also, we consider nonlinear systems affected by (unknown) disturbances $d$,

$$
\begin{gathered}
\dot{x}=f(x)+g(x) u+k(x) d, \\
\quad u \in \mathbb{R}^{m}, y \in \mathbb{R}^{p}, d \in \mathbb{R}^{q}, x \in M, \\
y=h(x), \quad f\left(x_{0}\right)=0, h\left(x_{0}\right)=0,
\end{gathered}
$$

where $k(x)$ is an $n \times q$ matrix, with entries depending smoothly on $x$. The corresponding linearization around $x_{0}$ is denoted as

$$
\dot{\bar{x}}=F \bar{x}+G \bar{u}+K \bar{d}, \quad \bar{u} \in \mathbb{R}^{m}, \bar{d} \in \mathbb{R}^{q}, \bar{x} \in \mathbb{R}^{n},
$$

$\bar{y}=H \bar{x}, \quad \bar{y} \in \mathbb{R}^{p}$,

where $F, G, H$ are defined in (4), while

$$
K=k\left(x_{0}\right) \text {. }
$$

The purpose of this note is to show that there is a close connection between the $H_{\infty}$ norm $\left(L_{2}\right.$-induced norm) of the linearized system (3) and some ' $H_{\infty}$ norm' of the nonlinear system (2). In fact we will prove that if (assuming $F$ to be asymptoti- 
cally stable and starting from the initial condition $\bar{x}=0$ )

$\|\bar{y}\|_{L_{2}}^{2}<\gamma^{2}\|\bar{u}\|_{L_{2}}^{2}$ for all $\bar{u} \in L_{2}(0, \infty)$

(i.e. the $H_{\infty}$ norm of the transfer matrix $H(I s-$ $F)^{-1} G$ of the linear system (3) is less than some constant $\gamma>0$ ), then also for the nonlinear system (2) we have (starting from the initial condition $\left.x(0)=x_{0}\right)$

$\|y\|_{L_{2}}^{2}<\gamma^{2}\|u\|_{L_{2}}^{2}$

for all $u \in L_{2}$ "such that $x$ is not driven too far from $x_{0}$ ". Here \|\|$_{L_{2}}$ denotes the usual $L_{2}$ norm, i.e.

$\|z\|_{L_{2}}^{2}=\int_{0}^{\infty} z^{\mathrm{T}}(t) z(t) \mathrm{d} t, \quad z: \mathbb{R}^{+} \rightarrow \mathbb{R}^{s}$.

Furthermore we will show that we have a similar relation between the standard state feedback $H_{\infty}$ control problem for the nonlinear system with disturbances (5) and its linearization (6). In fact we will prove that if we can find for (6) a stabilizing feedback $\bar{u}=L \bar{x}$ such that

$$
\sup _{\bar{d} \neq 0} \frac{\|\bar{y}\|_{L_{2}}^{2}+\|\bar{u}\|_{L_{2}}^{2}}{\|\bar{d}\|_{L_{2}}^{2}}<\gamma^{2}
$$

(i.e. the $H_{\infty}$ norm from disturbances to inputs and outputs is less than $\gamma$ ), then also for the nonlinear system we can find, at least locally around $x_{0}$, a nonlinear feedback $u=l(x)$ such that, if $x$ is not driven too far from $x_{0}$,

$\sup _{d \neq 0} \frac{\|y\|_{L_{2}}^{2}+\|u\|_{L_{2}}^{2}}{\|d\|_{L_{2}}^{2}}<\gamma^{2}$.

The main technical tool, which is of some interest in itself, is a lemma given in the next section which states that the stable invariant manifold of a Hamiltonian vector field with no imaginary eigenvalues is a Lagrangian submanifold, and so has a natural generating function attached to it. This generalizes the well-known fact that the generalized stable eigenspace of a Hamiltonian matrix having no imaginary eigenvalues corresponds to a symmetric solution of its related Riccati equation. Indeed, the generating function will enable us to use a 'completion of the squares' argument, which is very similar to the one used in the linear case (see e.g. [8]). For a discussion of the $H_{\infty}$ control problem for linear systems we refer to the lecture notes [7], while the recent state space approach to linear $H_{\infty}$ control can be found in $[6,8,11]$ and the references quoted therein.

\section{Stable manifolds of hyperbolic Hamiltonian vec- tor fields}

Consider a Hamiltonian vector field on a symplectic manifold $\left(N^{2 n}, \omega\right)$, with Hamiltonian $H: N^{2 n} \rightarrow \mathbb{R}$ and equilibrium $z_{0} \in N$,

$\dot{z}=X_{H}(z), \quad X_{H}\left(z_{0}\right)=0, \quad x \in N$.

By Darboux's theorem (see e.g. [1]) there exist local coordinates $\left(x_{1}, \ldots, x_{n}, p_{1}, \ldots, p_{n}\right)$ for $N$ such that

$\omega=\sum_{i=1}^{n} \mathrm{~d} p_{i} \wedge \mathrm{d} x_{i}$

and thus the equations take the well-known local form

$$
\begin{aligned}
& \dot{x}_{i}=\frac{\partial H}{\partial p_{i}}(x, p), \\
& \dot{p}_{i}=-\frac{\partial H}{\partial x_{i}}(x, p), \quad i=1, \ldots, n .
\end{aligned}
$$

Such coordinates are called canonical. Now suppose that $z_{0}$ is a hyperbolic equilibrium for $X_{H}$, i.e. the Jacobian matrix $\mathrm{D} X_{H}\left(z_{0}\right)$, given in canonical coordinates as

$\mathrm{D} X_{H}\left(z_{0}\right)=\left[\begin{array}{cc}\frac{\partial^{2} H}{\partial x \partial p} & \frac{\partial^{2} H}{\partial p^{2}} \\ -\frac{\partial^{2} H}{\partial x^{2}} & -\frac{\partial^{2} H}{\partial p \partial x}\end{array}\right]\left(z_{0}\right)$,

has no imaginary eigenvalues. Then by the Stable Manifold Theorem (see e.g. [1,7.2.3]) there exists a global invariant stable manifold $S$ through $x_{0}$ ( $S$ is an immersed submanifold of $N^{2 n}$ ), i.e. $S$ is invariant for the vector field $X_{H}$, and $X_{H}$ restricted to $S$ is asymptotically stable. Furthermore, $S$ is tangent at $z_{0}$ to the generalized stable eigenspace of $\mathrm{D} X_{H}\left(z_{0}\right)$, i.e.

$T_{z_{0}} S=X^{-}\left(\mathrm{D} X_{H}\left(z_{0}\right)\right)$

where $X^{-}\left(\mathrm{D} X_{H}\left(z_{0}\right)\right)$ is the $n$-dimensional eigenspace of the matrix $\mathrm{D} X_{H}\left(z_{0}\right)$ corresponding to its 
$n$ eigenvalues in the open left half-plane $\mathbb{C}^{-}$. (Recall that $\mathrm{D} X_{H}\left(z_{0}\right)$ is a Hamiltonian matrix, and thus its eigenvalues are symmetric with respect to the imaginary axis, see e.g. [1].)

A submanifold $L \subset\left(N^{2 n}, \omega\right)$ is called a Lagrangian submanifold (e.g. [1]) if $\operatorname{dim} L=n$ and furthermore $\omega$ restricted to $L$ is zero, i.e.

$\left.\omega\right|_{L}=0$.

Lemma 1. Suppose $z_{0}$ is a hyperbolic equilibrium for $X_{H}$ on $N^{2 n}$. Then the global stable invariant manifold $S$ of $X_{H}$ through $z_{0}$ is a Lagrangian submanifold of $N^{2 n}$.

Proof. By hyperbolicity and symmetry of the eigenvalues with respect to the imaginary axis, $\operatorname{dim} S=n$, so we only have to prove that $\omega$ restricted to $S$ is zero. Since $S$ is invariant for $X_{H}$ we can define $\bar{X}_{H}$ as the restriction of $X_{H}$ to $S$. Furthermore, let $\bar{\omega}$ be the restriction of $\omega$ to $S$. Since $X_{H}$ is Hamiltonian we have $L_{X_{H}}=0\left(L_{X_{H}}\right.$ denoting the Lie derivative w.r.t. $X_{H}$ ) and thus $L_{\bar{X}_{H}} \bar{\omega}=0$, or equivalently $\phi_{t}^{*} \bar{\omega}=\bar{\omega}$, where we denote by $\phi_{t}$ the flow of the vector field $\bar{X}_{H}$ on $S$. Hence for any $z \in S$ and $Z_{1}, Z_{2} \in T_{z} S$,

$\bar{\omega}(z)\left(Z_{1}, Z_{2}\right)=\bar{\omega}\left(\phi_{t}(z)\right)\left(\phi_{t}(z)_{*} Z_{1}, \phi_{t}(z)_{*} Z_{2}\right)$

for all $t \geq 0$. Now, since $\bar{X}_{H}$ is asymptotically stable,

$\phi_{t}(z){ }_{*} Z_{i} \rightarrow 0$ for $t \rightarrow \infty, \quad i=1,2$,

and thus $\bar{\omega}(z)\left(Z_{1}, Z_{2}\right)=0$.

Remark. Although the contents of the above lemma are plausible and its proof is simple I could not find any statement of it in the literature. Finally, we recall the following well-known fact from symplectic geometry (see e.g. [1]).

Proposition 2. Let $L \subset\left(N^{2 n}, \omega\right)$ be a Lagrangian submanifold, and suppose that in a neighborhood of some point $z_{0} \in L$ there exist centered (i.e. zero in $\left.z_{0}\right)$ canonical coordinates $\left(x_{1}, \ldots, x_{n}, p_{1}, \ldots, p_{n}\right)$, such that $L$ is parametrized by $x_{1}, \ldots, x_{n}$. Then there exists a function $V\left(x_{1}, \ldots, x_{n}\right)$ (possibly de- fined on a smaller neighbourhood of 0 ) such that $L$ is locally given as

$$
\begin{gathered}
\left\{\left(x_{1}, \ldots, x_{n}, p_{1}=\frac{\partial V}{\partial x_{1}}(x), \ldots,\right.\right. \\
\left.\left.p_{n}=\frac{\partial V}{\partial x_{n}}(x)\right) \mid x \text { around } 0 \in \mathbb{R}^{n}\right\} .
\end{gathered}
$$

$V$ is called a generating function of $L$ around $z_{0}$.

Combining Lemma 1 and Proposition 2 we obtain:

Proposition 3. Take the assumptions of Lemma 1, and assume that there exist canonical coordinates $\left(x_{1}, \ldots, x_{n}, p_{1}, \ldots, p_{n}\right)$ around $z_{0}$ such that $S$ is parametrized by $\left(x_{1}, \ldots, x_{n}\right)$. Then $S$ is locally of the form (20) for some generating function $V$. Furthermore, $V$ is a local solution of the HamiltonJacobi equation

$H\left(x, \frac{\partial V}{\partial x}(x)\right)=H\left(z_{0}\right), \quad x$ around $0 \in \mathbb{R}^{n}$.

Proof. We still we have to prove (21). Since $X_{\underline{H}}(H)=0, H$ is preserved by the flow of $\bar{X}_{H}$ ( $\bar{X}_{H}$ being the restriction of $X_{H}$ to $S$ ). Since $\bar{X}_{H}$ is asymptotically stable this means that for all $z \in S$,

$H(z)=H\left(\phi_{t}(z)\right) \rightarrow H\left(z_{0}\right)$ for $t \rightarrow \infty$

(with $\phi_{t}$ again denoting the flow of $\bar{X}_{H}$ ), and thus (21) follows since every $z \in S$ is of the form as given in (20).

Corollary 4. The symmetric matrix

$P_{V}:=\frac{\partial^{2} V}{\partial x^{2}}(0)$

(i.e. the Hessian matrix of $V$ in $x=0$ ) is the stabilizing solution of the algebraic Riccati equation (see e.g. [16])

$A^{\mathrm{T}} P+P A+P R P+Q=0$,

where we have set

$$
\begin{aligned}
& \frac{\partial^{2} H}{\partial x \partial p}(0,0)=: A, \quad \frac{\partial^{2} H}{\partial p^{2}}(0,0)=: R, \\
& \frac{\partial^{2} H}{\partial x^{2}}(0,0)=: Q .
\end{aligned}
$$


Proof. By (16) and (20) it immediately follows that

$\operatorname{span}\left[\begin{array}{c}I \\ P_{V}\end{array}\right]$

is the generalized stable eigenspace of the Hamiltonian matrix

$\left[\begin{array}{rc}A & R \\ -Q & -A^{\mathrm{T}}\end{array}\right]$

and thus $P_{V}$ defines the stabilizing solution of (22).

Remark. Note that (22) is the Hamilton-Jacobi equation corresponding to the quadratic Hamiltonian $p^{\mathrm{T}} A x+\frac{1}{2} p^{\mathrm{T}} R p+\frac{1}{2} q^{\mathrm{T}} Q q$, i.e. the quadratic term in the Taylor expansion of $H(x, p)$ around $(0,0)$.

\section{A nonlinear $L_{2}$-induced norm}

Let us now consider the nonlinear system (2) and its linearization (3). We make the following assumption.

Assumption 1. $F$ is asymptotically stable.

Then the $H_{\infty}$ norm of the transfer matrix $H(s I$ $-F)^{-1} G$ of (3) is defined, and we have the following basic observation in the state space approach to $H_{\infty}$ control $[6,5,2,16]$.

Lemma 5. Let $\gamma>0$ and let Assumption 1 be satisfied. Then

$\left\|H(s I-F)^{-1} G\right\|_{H_{\infty}}<\gamma$

if and only if there exists a symmetric solution $P$ to the Riccati equation

$F^{\mathrm{T}} P+P F+\frac{1}{\gamma^{2}} P G G^{\mathrm{T}} P+H^{\mathrm{T}} H=0$

satisfying

$\sigma\left(F+\frac{1}{\gamma^{2}} G G^{\mathrm{T}} P\right) \subset \mathbb{C}^{-}$

(i.e., $P$ is the stabilizing solution to the Riccati equation (25)).

We state our first main theorem.
Theorem 6. Consider the nonlinear system (2) and its linearization (3) satisfying Assumption 1. Let $\gamma>0$ be a constant.

Suppose that $\left\|H(s I-F)^{-1} G\right\|_{H_{\infty}}<\gamma$ (i.e. (8) is satisfied, see $[7,6])$. Then there exists a neighborhood $W$ of $x_{0}$ such that for all $u \in L_{2}(0, \infty)$ with compact support for which the solution $x\left(t, 0, x_{0}, u\right)$ of (2) remains in $W$ we have

$\|y\|_{L_{2}}^{2}<\gamma^{2}\|u\|_{L_{2}}^{2}$.

(Here, of course, $y(t)=h\left(x\left(t, 0, x_{0}, u\right)\right)$ denotes the output of (1).)

Proof. By Lemma 5 there exists a solution $P$ to (25) satisfying (26). Equivalently,

$\operatorname{span}\left[\begin{array}{l}I \\ P\end{array}\right]$

is the generalized stable eigenspace of the Hamiltonian matrix

$\operatorname{Ham}=\left[\begin{array}{cc}F & \frac{1}{\gamma^{2}} G G^{\mathrm{T}} \\ -H^{\mathrm{T}} H & -F^{\mathrm{T}}\end{array}\right]$.

In particular, Ham has no imaginary eigenvalues. Now, Ham is precisely the Jacobian matrix $\mathrm{D} X_{H}\left(x_{0}\right)$ of the Hamiltonian vector field $X_{H}$ on the symplectic manifold $N^{2 n}:=T^{*} M$ (endowed with its natural symplectic form, see [1]) corresponding to the Hamiltonian

$$
\begin{aligned}
H(x, p)= & p^{\mathrm{T}} f(x)+\frac{1}{2} \frac{1}{\gamma^{2}} p^{\mathrm{T}} g(x) g^{\mathrm{T}}(x) p \\
& +\frac{1}{2} h^{\mathrm{T}}(x) h(x)
\end{aligned}
$$

(where $(x, p)$ are the natural (and thus canonical) coordinates for $N=T^{*} M$ ). Hence $X_{H}$ has an $n$-dimensional stable invariant manifold $S$ passing through $z_{0}=\left(x_{0}, 0\right)$, which is Lagrangian by Lemma 1. Furthermore, by (16) and the fact that the generalized stable eigenspace of Ham is given in the form

$\operatorname{span}\left[\begin{array}{l}I \\ P\end{array}\right]$

it follows that $S$ is parametrized, locally around $z_{0}$, by the $x$-coordinates. Hence by Lemma $2, S$ is locally around $z_{0}$ of the form

$\left\{\left(x, \frac{\partial V}{\partial x}(x)\right) \mid x \in M, x\right.$ around $\left.x_{0}\right\}$ 
for some generating function $V$. Let now $W$ be a neighborhood of $x_{0}$ such that $W$ is in the domain of attraction of $x_{0}$ for $f$, and $V$ is defined on $W$. By Proposition 3 and the fact that $H\left(x_{0}, 0\right)=0$ we have

$$
\begin{aligned}
& \frac{\partial V}{\partial x}(x) f(x)+\frac{1}{2} \frac{1}{\gamma^{2}} \frac{\partial V}{\partial x}(x) g(x) g^{\mathrm{T}}(x)\left[\frac{\partial V}{\partial x}(x)\right]^{\mathrm{T}} \\
& \quad+\frac{1}{2} h^{\mathrm{T}}(x) h(x)=0
\end{aligned}
$$

Denoting $(\mathrm{d} / \mathrm{d} t) V$ as the derivative of $V$ along (2), we have by 'completing the squares'

$$
\begin{aligned}
\frac{\mathrm{d}}{\mathrm{d} t} V= & \frac{\partial V}{\partial x} f+\frac{\partial V}{\partial x} g u \\
= & -\frac{1}{2} \gamma^{2}\left\|u-\frac{1}{\gamma^{2}}\left[\frac{\partial V}{\partial x} g\right]^{\mathrm{T}}\right\|^{2} \\
& +\frac{\partial V}{\partial x} f+\frac{1}{2} \frac{1}{\gamma^{2}} \frac{\partial V}{\partial x} g g^{\mathrm{T}}\left[\frac{\partial V}{\partial x}\right]^{\mathrm{T}}+\frac{1}{2} \gamma^{2}\|u\|^{2},
\end{aligned}
$$

and thus by substituting (31), and denoting $h^{\mathrm{T}}(x) h(x)=\|y\|^{2}$,

$$
\begin{aligned}
\frac{\mathrm{d}}{\mathrm{d} t} V= & \frac{1}{2} \gamma^{2}\|u\|^{2}-\frac{1}{2}\|y\|^{2} \\
& -\frac{1}{2} \gamma^{2}\left\|u-\frac{1}{\gamma^{2}}\left[\frac{\partial V}{\partial x} g\right]^{\mathrm{T}}\right\|^{2},
\end{aligned}
$$

or

$$
\begin{aligned}
\|y\|_{L_{2}}^{2}-\gamma^{2}\|u\|_{L_{2}}^{2}= & 2 V\left(x_{0}\right)-2 V(x(\infty)) \\
& -\gamma^{2}\left\|u-\frac{1}{\gamma^{2}}\left[\frac{\partial V}{\partial x} g\right]^{\mathrm{T}}\right\|^{2}
\end{aligned}
$$

Since $u(t)=0$ for $t \geq T$ for some $T$, and the trajectories $x(t)$ remain in the domain of attraction of $x_{0}$ we have $x(\infty)=x_{0}$, and thus

$$
\|y\|_{L_{2}}^{2}-\gamma^{2}\|u\|_{L_{2}}^{2} \leq 0 \text {. }
$$

Hence it follows that $\|y\|_{L_{2}}^{2} \leq \gamma^{2}\|u\|_{L_{2}}^{2}$. To obtain strict inequality we observe that

$$
\left\|H(s I-F)^{-1} G\right\|_{H_{\infty}}<\gamma
$$

implies that there exists some $\tilde{\gamma}<\gamma$ such that $\left\|H(s I-F)^{-1} G\right\|_{H_{\infty}}<\tilde{\gamma}$. Repeating the same story for $\tilde{\gamma}$ we obtain

$$
\|y\|_{L_{2}}^{2} \leq \tilde{\gamma}^{2}\|u\|_{L_{2}}^{2}<\gamma^{2}\|u\|_{L_{2}}^{2} .
$$

Remark 1. Note that the above 'completion of the squares' argument is essentially the same as in the linear case $[8,16]$.

Remark 2. The condition on $u$ to be of compact support is only to ensure that $x(t) \rightarrow x_{0}$ for $t \rightarrow \infty$, since this is not a priori clear as in the linear case. Another possibility would be to require that $f$ and $g$ are globally Lipschitz continuous on $W$ (this is always true if $W$ is bounded!), since by [14,9] this implies that (setting $\left.x_{0}=0\right) \quad x \in L_{2}$ and $\dot{x} \in L_{2}$, and thus $x(t) \rightarrow 0$.

Corollary 7. Let Assumption 1 be satisfied, and let $\gamma>0$. Then (27) holds if $\left(x_{0}, 0\right)$ is a hyperbolic equilibrium for the Hamiltonian vector field corresponding to the Hamiltonian (29).

Proof. Hyperbolicity is equivalent to the property that the Hamiltonian matrix Ham defined in (28) has no imaginary eigenvalues. It is well-known in the theory of Riccati equations (see e.g. [6, Lemma 4]) that, since $(F, G)$ is stabilizable by Assumption 1, this implies the existence of a symmetric solution $P$ to (25) satisfying (26). Therefore by Lemma 5 ,

$$
\left\|H(s I-F)^{-1} G\right\|_{H_{\infty}}<\gamma,
$$

and the result follows from Theorem 6 .

4. The nonlinear state feedback $H_{\infty}$ control problem

Now we come to our main theorem on the standard $H_{\infty}$ control problem using state feedback. For the linearized system with disturbances (6) we consider the $H_{\infty}$ norm of the transfer matrix

$T_{L}: \bar{d} \rightarrow z=\left(\begin{array}{l}\bar{y} \\ \bar{u}\end{array}\right)$ 
of the closed-loop system

$\dot{\bar{x}}=F \bar{x}+G \bar{u}+K \bar{d}$,

$\bar{y}=H \bar{x}$,

$\bar{u}=L \bar{x}$,

for any stabilizing state feedback $\bar{u}=L \bar{x}$, i.e.

$F+G L$ is asymptotically stable.

Thus for any $L$ satisfying (35) we consider

$\left\|T_{L}\right\|_{H_{\infty}}^{2}:=\sup _{\bar{d} \neq 0} \frac{\|\bar{y}\|_{L_{2}}^{2}+\|\bar{u}\|_{L_{2}}^{2}}{\|\bar{d}\|_{L_{2}}^{2}}$.

We recall the following theorem from linear $H_{\infty}$ control (see $[6,11,15])$.

Assumption 2. $(F, G)$ is stabilizable, and $(H, F)$ is detectable.

Theorem 8. Let Assumption 2 be satisfied for the linearized system (6) and let $\gamma>0$. Then

$\inf _{L \text { satisfying (35) }}\left\|T_{L}\right\|_{H_{\infty}}<\gamma$

if and only if there exists a symmetric solution $P \geq 0$ of

$F^{\mathrm{T}} P+P F-P\left(G G^{\mathrm{T}}-\frac{1}{\gamma^{2}} K K^{\mathrm{T}}\right) P+H^{\mathrm{T}} H=0$,

satisfying

$\sigma\left(F-G G^{\mathrm{T}} P+\frac{1}{\gamma^{2}} K K^{\mathrm{T}} P\right) \subset \mathbb{C}^{-}$.

Furthermore one possible $L$ satisfying (35) and $\left\|T_{L}\right\|_{H_{\infty}}<\gamma$ is given by

$L=-G^{\mathrm{T}} P$.

For the nonlinear system (5) we obtain our second main theorem:

Theorem 9. Consider the nonlinear system (5). Let Assumption 2 be satisfied for its linearization (6). Let $\gamma>0$. Suppose that (37) is satisfied for (6).
Then there exists a neighborhood $W$ of $x_{0}$ and a nonlinear feedback $u=l(x)$ such that

$\dot{x}=f(x)+g(x) l(x)$ is globally

asymptotically stable on $W$,

$\|y\|_{L_{2}}^{2}+\|u\|_{L_{2}}^{2}<\gamma^{2}\|d\|_{L_{2}}^{2}$,

for all disturbance functions $d \in L_{2}$ with compact support such that the state space trajectories starting from $x(0)=x_{0}$ remain in $W$.

Proof. Consider the Hamiltonian vector field $X_{H}$ on $N^{2 n}=T^{*} M$ corresponding to the Hamiltonian

$$
\begin{array}{rl}
H(x, p)=p^{\mathrm{T}} & f(x)-\frac{1}{2} p^{\mathrm{T}}\left(g(x) g^{\mathrm{T}}(x)\right. \\
& \left.\quad-\frac{1}{\gamma^{2}} k(x) k^{\mathrm{T}}(x)\right) p+\frac{1}{2} h^{\mathrm{T}}(x) h(x) .
\end{array}
$$

By Theorem 8 there exists a stabilizing solution $P$ to (38), or equivalently

$\operatorname{span}\left[\begin{array}{l}I \\ P\end{array}\right]$

is the generalized stable eigenspace of

$\operatorname{Ham}=\left[\begin{array}{cc}F & -G G^{\mathrm{T}}+\frac{1}{\gamma^{2}} K K^{\mathrm{T}} \\ -H^{\mathrm{T}} H & -F^{\mathrm{T}}\end{array}\right]$.

Since Ham $=\mathrm{D} X_{H}\left(z_{0}\right), z_{0}=\left(x_{0}, 0\right)$, we conclude that $X_{H}$ has an $n$-dimensional stable invariant manifold $S$ passing through $z_{0}$, which is Lagrangian by Lemma 1 and which is parametrized, locally around $z_{0}$, by the $x$-coordinates. Hence by Lemma 2, locally around $z_{0}, S$ is given as

$S=\left\{\left(x, \frac{\partial V}{\partial x}(x)\right) \mid x \in M, x\right.$ around $\left.x_{0}\right\}$

for some generating function $\boldsymbol{V}$. By Proposition 3 and $H\left(x_{0}, 0\right)=0$ we have

$$
\begin{aligned}
& \frac{\partial V}{\partial x}(x) f(x) \\
& -\frac{1}{2} \frac{\partial V}{\partial x}(x)\left(g(x) g^{\mathrm{T}}(x)-\frac{1}{\gamma^{2}} k(x) k^{\mathrm{T}}(x)\right) \\
& \cdot\left[\frac{\partial V}{\partial x}(x)\right]^{\mathrm{T}}+\frac{1}{2} h^{\mathrm{T}}(x) h(x)=0 .
\end{aligned}
$$


Denote $(\mathrm{d} / \mathrm{d} t) V$ as the derivative of $V$ along (5). Then by 'completing the squares', and using (46),

$$
\begin{aligned}
\frac{\mathrm{d}}{\mathrm{d} t} V= & \frac{\partial V}{\partial x} f+\frac{\partial V}{\partial x} g u+\frac{\partial V}{\partial x} k d \\
= & \frac{1}{2}\left\|u+\left[\frac{\partial V}{\partial x} g\right]^{\mathrm{T}}\right\|^{2} \\
& -\frac{1}{2} \gamma^{2}\left\|d-\frac{1}{\gamma^{2}}\left[\frac{\partial V}{\partial x} k\right]^{\mathrm{T}}\right\|^{2} \\
& -\frac{1}{2}\|u\|^{2}+\frac{1}{2} \gamma^{2}\|d\|^{2} \\
& +\frac{\partial V}{\partial x} f-\frac{1}{2} \frac{\partial V}{\partial x} g g^{\mathrm{T}}\left[\frac{\partial V}{\partial x}\right]^{\mathrm{T}} \\
& +\frac{1}{2} \frac{1}{\gamma^{2}} \frac{\partial V}{\partial x} k k^{\mathrm{T}}\left[\frac{\partial V}{\partial x}\right]^{\mathrm{T}} \\
= & \frac{1}{2}\left\|u+\left[\frac{\partial V}{\partial x} g\right]^{\mathrm{T}}\right\|^{2} \\
& -\frac{1}{2} \gamma^{2}\left\|d-\frac{1}{\gamma^{2}}\left[\frac{\partial V}{\partial x} k\right]^{\mathrm{T}}\right\|^{2} \\
& -\frac{1}{2}\|y\|^{2}-\frac{1}{2}\|u\|^{2}+\frac{1}{2} \gamma^{2}\|d\|^{2} .
\end{aligned}
$$

Choosing the state feedback

$u=-\left[\frac{\partial V}{\partial x}(x) g(x)\right]^{\mathrm{T}}=: l(x)$

we thus obtain

$$
\begin{aligned}
& \|y\|_{L_{2}}^{2}+\|u\|_{L_{2}}^{2}-\gamma^{2}\|d\|_{L_{2}}^{2} \\
& \quad=2 V\left(x_{0}\right)-2 V(x(\infty))-\gamma^{2}\left\|d-\frac{1}{\gamma^{2}}\left[\frac{\partial V}{\partial x} k\right]^{\mathrm{T}}\right\|^{2} .
\end{aligned}
$$

Since the solution $P$ of (38), (39) equals the Hessian matrix $\left(\partial^{2} V / \partial x^{2}\right)\left(x_{0}\right)$ (compare with Corollary 4$)$, while $(\partial V / \partial x)\left(x_{0}\right)=0$ by the fact that $z_{0}=\left(x_{0}, 0\right)$, we observe from (48) that

$$
\frac{\partial l}{\partial x}\left(x_{0}\right)=L
$$

with $L$ defined in (40). Thus by Theorem 8 the vector field $f(x)+g(x) l(x)$ is locally exponentially stable around $x_{0}$. It is now clear how to choose $W$. Indeed $W$ has to be contained in the domain of definition of $V$ and in the domain of attraction of the vector field $\dot{x}=f(x)+g(x) l(x)$.
Then for $d$ as in the formulation of the theorem, $x(\infty)=x_{0}$, and thus

$\|y\|_{L_{2}}^{2}+\|u\|_{L_{2}}^{2} \leq \gamma^{2}\|d\|_{L_{2}}^{2}$.

Similarly as in the end of the proof of Theorem 6 we get strict inequality, and thus (42) follows.

Corollary 10. Let Assumption 2 and (37) be satisfied for (6). Then locally around $x_{0}$ there exists a unique solution $V$ of the Hamilton-Jacobi equation (46), satisfying the boundary conditions

$V\left(x_{0}\right)=0, \quad \frac{\partial V}{\partial x}\left(x_{0}\right)=0, \quad \frac{\partial^{2} V}{\partial x^{2}}\left(x_{0}\right)=P$,

where $P \geq 0$ is the unique solution of (25), (26). Furthermore, a feedback $u=l(x)$ such that (41) and (42) hold for some neighborhood $W$ of $x_{0}$ is given as

$l(x):=-\left[\frac{\partial V}{\partial x}(x) g(x)\right]^{\mathrm{T}}$.

Moreover if (46), (51) has a global solution $V$ on $M=\mathbb{R}^{n}$ such that $V$ is a positive definite function on $\mathbb{R}^{n}$ (in the sense of [13]), while the set

$\left\{x \in \mathbb{R}^{n} \mid \frac{\partial V}{\partial x}(x)(f(x)+g(x) l(x))=0\right\}$

contains no non-trivial trajectories of $\dot{x}=f(x)+$ $g(x) l(x)$ (with $l(x)$ as above), then we can take $W=\mathbb{R}^{n}$ (and thus we obtain a global solution to the nonlinear $H_{\infty}$ optimal control problem).

Proof. The first part is just a restatement of Theorem 9. Indeed if $V$ is a local solution of (46), (51) then

$$
L:=\left\{\left(x, \frac{\partial V}{\partial x}(x)\right) \mid x \text { around } x_{0}\right\}
$$

equals the stable invariant manifold $S$ of $X_{H}$. In fact, since $H=0$ on $L$ and $L$ is Lagrangian, $L$ is invariant for $X_{H}$ ([1, Prop. 5.3.32]). Furthermore by (51), $L$ is tangent at $z_{0}=\left(x_{0}, 0\right)$ to the generalized stable eigenspace of Ham defined in (44).

If $V$ is globally defined and satisfying the above conditions then global asymptotic stability follows from [13, Theorem 87] by consideration of (47), and thus we can take $W=\mathbb{R}^{n}$.

Remark 1. Analogously to the Remark after Theorem 6 we can replace the compact support condi- 
tion on the disturbance $d$ by a global continuity condition on the vector fields $f, g_{j}$ and $k_{j}$.

Remark 2. It follows from (47) that we cannot decrease $\gamma$ in (42) by allowing for dynamic nonlinear state feedback (just as in the linear case, see [11]).

Remark 3. One observes from (49) that

$d=\frac{1}{\gamma^{2}}\left[\frac{\partial V}{\partial x}(x) k(x)\right]^{\mathrm{T}}$

is the 'worst case' disturbance.

Remark 4. Notice that for $\gamma \rightarrow \infty$ the HamiltonJacobi equation (46) tends to the HamiltonJacobi-Bellman equation of the optimal control problem for the cost functional $\frac{1}{2}\|y\|_{L_{2}}^{2}+\frac{1}{2}\|u\|_{L_{2}}^{2}$ (compare with the linear case $[6,15]$ ).

Finally we remark that, like in the linear case (see e.g. $[5,6]$ ), the infimal $\gamma$ such that (42) holds to be computed by some iterative procedure.

\section{Conclusions}

It is clear that only a few first steps have been taken towards a state space approach of ' $H_{\infty}$ control' for nonlinear systems. In particular it would be desirable to have some a priori estimate of the size of the neighborhood $W$ in Theorem 9. Also, in this paper only the state feedback case has been considered, without entering the much more complicated dynamic output feedback case. It should be also of interest to make comparisons with recent advances in the operator and game theoretic approach to nonlinear $H_{\infty}$ control (see e.g. $[3,4]$ ), and with older work on (input-output) stability for nonlinear systems (see e.g. $[9,13,14]$ ).

\section{Acknowledgement}

I would like to thank S. Weiland for a very useful discussion.

\section{References}

[1] R.A. Abraham and J.E. Marsden, Foundations of Mechanics, 2nd edition (Benjamin/Cummings, Reading. MA, 1978).

[2] B.D.O. Anderson, An algebraic solution to the spectral factorization problem, IEEE Trans. Automat. Control 12 (1967) 410-414.

[3] J.A. Ball and J.W. Helton, Factorization of nonlinear systems: Towards a theory for nonlinear $H^{\infty}$ control, 27 th IEEE CDC, Austin, TX (1988) 2376-2381.

[4] J.A. Ball and J.W. Helton, $H^{\infty}$ control for nonlinear plants: connections with differential games, 28th IEEE CDC, Tampa, FL (1989) 956-962.

[5] B. Boyd, V. Balakrishnan and P. Kabamba, A bisection method for computing the $H_{\infty}$ norm of a transfer matrix and related problems, Math. Control Signals and Systems 2 (1989) 207-220.

[6] J.C. Doyle, K. Glover, P.P. Khargonekar and B.A. Francis, State-space solutions to standard $H_{2}$ and $H_{\infty}$ control problems, IEEE Trans. Automat. Control 34 (1990) 831846.

[7] B.A. Francis, $A$ Course in $H_{\infty}$ Control Theory, LNCIS 88 (Springer-Verlag, Berlin-New York, 1987).

[8] K. Glover and J.C. Doyle, A state space approach to $H_{\infty}$ optimal control, in: H. Nijmeijer, J.M. Schumacher, Eds., Three Decades of Mathematical System Theory, LNCIS 135 (Springer-Verlag, Berlin-New York, 1989) 179-218.

[9] D.J. Hill and D.J. Moylan, Connections between finite gain and asymptotic stability, IEEE Trans. Automat. Control 25 (1980) 931-936.

[10] A. Isidori, Nonlinear Control Systems, 2nd edition (Springer-Verlag, Berlin-New York, 1989).

[11] P.P. Khargonekar, I.R. Petersen and M.A. Rotea, $H^{\infty}$ optimal control with state feedback, IEEE Trans. Automat. Control 33 (1988) 786-788.

[12] H. Nijmeijer and A.J. van der Schaft, Nonlinear Dynamical Control Systems (Springer-Verlag, Berlin-New York, 1990).

[13] M. Vidyasagar, Nonlinear Systems Analysis (Prentice-Hall, Englewood Cliffs, NJ, 1978).

[14] M. Vidyasagar and A. Vanelli, New relationships between input-output and Lyapunov stability, IEEE Trans. Automat. Control 27 (1982) 481-483.

[15] S. Weiland, Ph.D. Thesis, University of Groningen (1990, to appear).

[16] J.C. Willems, Least-squares stationary optimal control and the algebraic Riccati equation, IEEE Trans. Automat. Control 16 (1971) 621-634. 